\title{
PSICOEDUCAÇÃO DE PROFESSORAS: CONTRIBUIÇÕES DA TEORIA COGNITIVO-COMPORTAMENTAL PARA PROMOÇÃO DE SAÚDE MENTAL NO CONTEXTO ESCOLAR
}

\section{Teachers' Psichoeducation: Contributions of Cognitive Behavioral Theory to promote mental health in school}

\begin{abstract}
Sabrina Torres Gomes - Universidade Federal do Recôncavo da Bahia/Brasil Fernanda Conceição de Jesus - Universidade Federal do Recôncavo da Bahia/Brasil Lusimara Gonçalves Miranda da Silva Jesus - Universidade Federal do Recôncavo da Bahia/Brasil
\end{abstract}

\begin{abstract}
RESUMO: O presente artigo relata a experiência vivenciada através da coordenação do projeto de extensão: Educação Psicoemocional de Professores(as), como parte das atividades desenvolvidas no Centro de Formação de Professores da Universidade Federal do Recôncavo da Bahia (CFP/UFRB). O projeto teve como objetivo trabalhar habilidades de autoconhecimento, autocontrole e desenvolvimento de habilidades socioemocionais em professores e professoras em formação através da Psicoeducação sobre emoções. $\mathrm{O}$ grupo de participantes foi composto por catorze mulheres, doze estudantes do curso de pedagogia, duas professoras recém formadas e duas monitoras do projeto (também estudantes de pedagogia), além da coordenadora. As atividades aconteceram quinzenalmente ao longo de um semestre letivo, tendo sido organizadas em oficinas temáticas sobre as emoções e temas correlatos. Os resultados indicaram a necessidade de promover com maior frequência atividades capazes de proporcionar informações sobre autoconhecimento para professores (as) em formação, que extrapolem os conteúdos teórico-práticos trabalhados durante a graduação, e possam ao mesmo tempo contribuir para a formação humana subjacente à prática profissional, contribuindo para o contato com a realidade escolar de maneira saudável e eficaz.
\end{abstract}

Palavras-chave: Formação de Professores. Psicoeducação. Teoria CognitivoComportamental (TCC).

ABSTRACT: This article reports the experience lived through the coordination of the extension project: Teachers' Psycho-Emotional Education, as part of the activities developed at the Teacher Training Center of the Federal University of Recôncavo da Bahia (CFP / UFRB). The project aimed to work self-knowledge skills, self-control and development of socio-emotional skills in teachers in formation through psychoeducation about emotions. The group of participants consisted of fourteen women, twelve pedagogy students, two newly graduated teachers and two project supervisors (also pedagogy students), as well as the coordinator. The activities took place biweekly during a semester, and were organized in thematic workshops on emotions and related topics. The results indicated the need to promote more frequently activities capable of providing information about self-knowledge to undergraduate teachers, which go beyond the theoretical and practical contents worked during the undergraduate course and at the same time

Educação, Psicologia e Interfaces, Volume 3, Número3, p. 94-106, Setembro/Dezembro, 2019.

ISSN: 2594-5343. DOI: https://doi.org/10.37444/issn-2594-5343.v3i3.173 
contribute to the human formation underlying the practice contributing to the contact with the school reality in a healthy and effective way.

Keywords: Teacher training. Psychoeducation. Cognitive Behavioral Theory.

\section{INTRODUÇÃO}

É cada vez mais crescente a quantidade de pesquisas que investigam as relações entre as práticas docentes no ambiente escolar e o adoecimento dos profissionais da educação. Sobrecarga de trabalho, baixos salários, formação precária, responsabilização exclusiva por fracassos ou dificuldades no desempenho de suas atividades em sala de aula, ausência ou escassez de material, são alguns dos fatores com os quais professores e professoras precisam lidar no seu dia a dia laboral nas escolas e Universidades. Esses fatores, somados ao excesso de demanda, são considerados gatilhos que contribuem para o aumento do estresse relacionado ao trabalho podendo, portanto, levar ao adoecimento não somente físico como psíquico (CRUZ et al. 2010; OMS, 2008).

O excesso de cobranças não é exclusivo à categoria de professores, mas sim uma exigência do mundo contemporâneo que tem levado as pessoas a respostas comportamentais nem sempre adaptativas. No entanto, excetuando-se os profissionais cujas atividades envolvem risco de vida como, médicos, bombeiros e enfermeiros, por exemplo, os professores aparecem no topo da lista quando se tratam dos efeitos nefastos do contexto de trabalho sobre a saúde mental de trabalhadores. Dentre as doenças psíquicas que mais comumente acometem esses profissionais destacam-se a depressão, a ansiedade e a chamada síndrome de burnout, esta última caracterizada pelo esgotamento emocional gerado pelas relações com o trabalho. Ademais, é importante destacar que esse tipo de adoecimento não depende somente das características do sujeito, mas também de fatores socioambientais desencadeantes, tais como aqueles descritos anteriormente (CARLOTTO, 2014; CRUZ et al. 2010).

Na realidade das escolas públicas, as condições de trabalho que levam a ausências frequentes e/ou afastamento de professores por motivos de adoecimento mental, como os aqui apresentados, são ainda mais evidentes e preocupantes, sendo estas de notório conhecimento. De acordo com Castro et al. (2003), o adoecimento psíquico em professores, especialmente pela síndrome de burnout, tem levado a maiores repercussões

Educação, Psicologia e Interfaces, Volume 3, Número3, p. 94-106, Setembro/Dezembro, 2019.

ISSN: 2594-5343. DOI: https://doi.org/10.37444/issn-2594-5343.v3i3.173 
devido aos danos causados direta e indiretamente, não somente sobre suas atividades e desajustes perante as atribuições laborais, mas também sobre a qualidade de suas relações com o ambiente educacional, chegando a repercutir diretamente na aprendizagem dos estudantes.

Conhecendo de perto a realidade da atuação docente em diversos níveis da escolarização, atuando como professora Universitária, ministrando aulas para os cursos de licenciatura do Centro de Formação de Professores (CFP) da Universidade Federal do Recôncavo da Bahia (UFRB) e, entendendo que o adoecimento psíquico pode ser minimizado ou evitado a partir de intervenções específicas da psicologia, foi que surgiu a proposta de desenvolver um projeto de extensão voltado para a promoção e prevenção da saúde mental de professores(as) em formação. O que pode ser notado no contato com esses(as) estudantes é um despreparo, inicialmente pessoal, mas principalmente profissional, para lidar com a realidade do contexto escolar dentro do sistema educacional vigente, indicando a necessidade de promover ações que extrapolem as discussões e práticas conteudistas e busquem trabalhar os fatores humanos que caracterizam as pessoas por detrás do seu exercício e identidade profissional.

Essas afirmações reforçam o crescente reconhecimento da necessidade de ampliar o olhar para as questões docentes que não somente possam melhorar as condições de saúde mental desses trabalhadores, mas que também possam repercutir em práticas mais efetivas no contexto escolar que repercutam também nas condições de aprendizagem para os estudantes (MELLO; CORREIA JUNIOR, 2018). A apropriação desse lugar tem estado relacionada ao papel das emoções no contexto educacional. Atualmente há a compreensão de que não somente é possível trabalharmos as nossas emoções, mas também devemos fazê-lo para que estejamos aptos a lidar com as inúmeras exigências da contemporaneidade de maneira adequada e funcional (CAMINHA, 2014).

O interesse crescente em trabalhar a saúde mental no contexto escolar tem sua origem numa série de fatores sócio-econômicos que abriram as portas do século XXI apresentando adoecimentos "invisíveis" como importantes marcadores da nossa época. Começou-se a perceber que o excesso de exigências e as atribuições de responsabilidades cada vez mais individualizadas, tanto em relação ao fracasso quanto ao sucesso dos sujeitos, trouxe consigo um aumento significativo na incidência de doenças como ansiedade, depressão, fobia social e síndrome de burnout (SILVA et al. 2009). Além de

Educação, Psicologia e Interfaces, Volume 3, Número3, p. 94-106, Setembro/Dezembro, 2019. 
serem sintomas de um adoecimento de ordem psicossocial, a elevada incidência de doenças dessa natureza entre professores e professoras deve servir de alerta para o desenvolvimento de estratégias capazes de preveni-las.

Essas constatações têm refletido em discussões frequentes sobre a relevância do trabalho do psicólogo junto à comunidade escolar, abrindo espaço para que a psicologia traga contribuições contextualizadas com as demandas da contemporaneidade, não somente no aspecto teórico, didático ou comportamental, mas como ferramenta de acesso ao autoconhecimento e mudanças pessoais que levem os sujeitos sociais a aprenderem a lidar com um ambiente cada vez mais estressor e adoecedor de maneiras mais funcionais e, portanto, mais adaptativas. Apesar das diversas teorias psicológicas compreenderem a inseparável relação entre sujeito e ambiente para a explicação dos fenômenos psíquicos, o modelo cognitivo-comportamental tem se destacado nesse sentido por enfatizar a força das crenças na avaliação que as pessoas fazem de si, do mundo e do futuro, demonstrando a influência direta sobre suas emoções, pensamentos e comportamentos (WRIGHT; BASCO; THASE, 2008).

Tendo em vista os aspectos destacados até aqui, esse artigo apresentará um relato de experiência a partir da realização do projeto de extensão: Educação Psicoemocional de Professores (as), desenvolvido pela Universidade Federal do Recôncavo da Bahia (UFRB) no Centro de Formação de Professores (CFP) no município de Amargosa-Ba. Esse projeto teve como objetivo trabalhar habilidades de autoconhecimento, autocontrole e desenvolvimento de habilidades socioemocionais junto a um grupo de professoras em formação (estudantes do curso de licenciatura da UFRB) e egressas, através da Psicoeducação das emoções, usando como referencial teórico-prático a Teoria CognitivoComportamental (TCC).

O modelo da Psicoeducação parte do princípio de que o adoecimento humano em seu sentido amplo, não ocorre de maneira isolada, nem parte "de dentro para fora", mas é fruto de uma construção que cada pessoa tem de si mesma, abrindo espaço para o trabalho de orientação e prevenção sobre vivências comumente adoecedoras, de maneira responsável e contextualizada. Nesse cenário, encontram-se alguns estudos voltados para o trabalho com professores (as) cujo recurso utilizado é a Psicoeducação. Em poucas palavras, psicoeducar implica em ensinar as pessoas a observarem seus comportamentos, 
pensamentos e emoções a partir da concepção de sujeito integral e em constante relação com o mundo (LEMES; ONDERE NETO, 2017; NOGUEIRA et al. 2017).

\section{MATERIAIS E MÉTODOS DA EXPERIÊNCIA}

A experiência vivenciada através do projeto Educação Psicoemocional de Professores(as) ocorreu quinzenalmente, ao longo de um semestre letivo em uma das instalações do CFP/UFRB, no município de Amargosa-Ba. As atividades foram planejadas a partir da compreensão de que nos cursos de licenciatura, especialmente no curso de pedagogia, tem havido poucas ou simplesmente não existem, práticas voltadas para trabalhar esse futuro profissional para que possa lidar com o cotidiano da realidade escolar. O reconhecimento dessa fragilidade que está presente também na sensação de incapacidade para lidar com um universo de cobranças e adversidades do contexto escolar, como falta de materiais e recursos limitados, crianças que sofrem abuso físico ou psicológico, pouca higiene ou educação doméstica, dentre outros aspectos, o trabalho para uma formação humanizada de professores e professoras torna-se imprescindível.

Considerando as questões aventadas até aqui, as atividades foram organizadas de acordo com dois eixos: trabalho com o grupo de extensão formado por mim, coordenadora do projeto, e pelas monitoras e, Psicoeducação de professoras em formação e recém egressas. O planejamento das atividades e a preparação teórica do grupo de extensão para a realização do projeto tiveram início um mês antes do início das atividades de Psicoeducação junto ao segundo eixo. Essa etapa foi fundamental para a elaboração e adequação das atividades de Psicoeducação voltadas à realidade das professoras em formação do CFP/UFRB, pois os autores e materiais utilizados na sua organização advêm da Teoria Cognitivo-Comportamental, com origens e aplicabilidades essencialmente psicológicas, é desconhecida da maioria dos(as) estudantes dos cursos de licenciatura, inclusive da pedagogia.

O projeto foi divulgado através de redes sociais e nas salas de aula, entre os(as) estudantes do CFP/UFRB, contendo as informações sobre tema, objetivo, início, local e horários dos encontros, na intenção de formar um grupo voluntário de professores(as) em formação interessado em desenvolver o autoconhecimento. Após o encerramento das inscrições, entramos em contato com as interessadas e confirmamos o primeiro encontro. Compreendendo que o exercício da atividade docente exige inúmeras habilidades e 
Psicoeducação de professoras: contribuições da Teoria Cognitivo-Comportamental para promoção de saúde mental no contexto escolar

adaptações, conforme já discutido anteriormente, trabalhamos alguns temas que consideramos fundamentais para o desenvolvimento do autoconhecimento e suas relações com as práticas docentes, através da Psicoeducação usando como referência os conceitos centrais da TCC para cada um deles através de oficinas realizadas quinzenalmente.

As oficinas, que aqui vamos chamar de oficinas psicoeducativas, foram organizadas de modo que pudessem proporcionar aprendizagem progressiva e continuada, de acordo com a ordem apresentada a seguir: Conhecendo a si mesmo e ao outro; Educação emocional positiva; Desenvolvendo a empatia; Trabalhando a Assertividade; e Promovendo o autoconhecimento. A cada encontro as atividades eram divididas em quatro momentos, respeitando a seguinte ordem: exercícios de MindfulnessI (atenção plena); Questionamento sobre o conhecimento que tinham em relação ao tema, seguido da apresentação e discussão sobre o assunto que incluía sua definição e os comportamentos, emoções e pensamentos a ele relacionados; Uma atividade prática para ser realizada em equipes e depois discutida com todo o grupo; e avaliação da experiência, relevância do tema e da aprendizagem.

A cada encontro, procuramos retomar a temática da semana anterior, na intenção de construir conjuntamente a concepção de sujeito integral e, portanto, inseparável de si mesmo e do contexto, promovendo reflexões sobre nossos próprios comportamentos cotidianos e sobre a influência que estes sofrem tanto da maneira como lidamos com as nossas emoções quanto dos nossos comportamentos. É importante destacar que, apesar do uso de recursos teóricos e práticos advindos originalmente da TCC, o projeto aqui apresentado não teve como proposta qualquer intervenção com fins psicoterapêuticos, utilizando como referência para a realização das atividades os princípios da Psicoeducação.

\section{RESULTADOS E DISCUSSÃO}

Os resultados da experiência aqui relatada elucidaram algumas necessidades emergentes e, em certo sentido urgentes, no que diz respeito à formação de professores e professoras, assim como trouxeram novas perspectivas para as contribuições da psicologia nesse processo. Nesse sentido, o papel da psicologia na educação extrapola as questões que dizem respeito às relações entre desenvolvimento e aprendizagem, olhando

Educação, Psicologia e Interfaces, Volume 3, Número3, p. 94-106, Setembro/Dezembro, 2019.

ISSN: 2594-5343. DOI: https://doi.org/10.37444/issn-2594-5343.v3i3.173 
o contexto educacional para além dos estudantes, propondo ações preventivas e formadoras de sujeitos mais capazes para lidar com a diversidade do mundo contemporâneo.

Os fundamentos da TCC buscam compreender a influência do ambiente, mais especificamente da interpretação que as pessoas fazem dos eventos que ocorrem nele, nas emoções, pensamentos e comportamentos dos sujeitos. Essas assertivas passam pela compreensão de que algumas interpretações levam a pensamentos disfuncionais, o que, nesses casos, produz, fortalece ou retroalimenta o adoecimento mental (WRIGHT; BASCO; THASE, 2008). Sendo assim, é possível compreender a expansão das práticas da TCC, que surgiram no contexto da psicologia clínica, para outras áreas de atuação, mostrando-se uma abordagem em constante atualização não apenas teórica, mas principalmente empírica.

Atualmente existem alguns programas baseados nos princípios e recursos da TCC voltados para grupos externos ao ambiente terapêutico, como é o caso da orientação de pais, do TRI preventivo no contexto escolar e das intervenções voltadas para melhorias na aprendizagem de estudantes (ALVES, 2018). Essas novas práticas não ocorrem por acaso, mas advêm da constatação da necessidade de trabalhar a saúde mental in loco, ampliando as possibilidades de atuação profissional das psicólogas de maneira contextualizada. No entanto, ainda são incipientes os programas voltados para professores, como se pode verificar nos elevados índices de adoecimento dessa categoria profissional, apesar do conhecimento acerca dos processos promotores do seu adoecimento psíquico ser amplamente documentado (SILVA, 2006; ANDRADE; CARDOSO, 2012).

Nesse cenário, as experiências vivenciadas a partir das atividades do projeto aqui apresentado procuraram psicoeducar futuras educadoras e educadoras recém formadas sobre os principais fatores de adoecimento psíquico, promovendo as oficinas em caráter preventivo. As análises realizadas a partir das avaliações feitas após cada oficina e também no final dessa primeira etapa do projeto foram divididas de acordo com os dois eixos anteriormente apresentados. Notou-se que para todas as participantes, independente dos referidos eixos, as assertivas de Silva (2006) e de Andrade e Cardoso (2012) foram corroboradas pela experiência vivenciada durante o projeto, indicando ao mesmo tempo

Educação, Psicologia e Interfaces, Volume 3, Número3, p. 94-106, Setembro/Dezembro, 2019.

ISSN: 2594-5343. DOI: https://doi.org/10.37444/issn-2594-5343.v3i3.173 
Psicoeducação de professoras: contribuições da Teoria Cognitivo-Comportamental para promoção de saúde mental no contexto escolar

a falta de conhecimento sobre as causas do adoecimento e a necessidade de trabalhar as questões a ele.

As participantes voluntárias consideraram que as temáticas trabalhadas nas oficinas psicoeducativas foram de ampla relevância para suas construções pessoais, trazendo contribuições em inúmeros aspectos de suas vidas. Para elas, a forma como os temas foram organizados e trabalhados ao longo do processo facilitou a compreensão de conceitos do cotidiano, a exemplo das emoções e da empatia, a partir de novos olhares, abrindo perspectivas de autoconhecimento e mudança de comportamentos antes desconhecidas pelas pessoas desse grupo. Os resultados de Silva e Silva (2009) sobre o conhecimento de professores a respeito da educação emocional indicaram noções incipientes sobre Inteligência Emocional além de identificar que cerca de $10 \%$ dos participantes desconhecem qualquer informação sobre o tema. Esses dados reforçam as avaliações do projeto aqui relatado, demonstrando que o contexto escolar necessita de mais esforços para ultrapassar seu histórico conteudista, promovendo o desenvolvimento dos recursos humanos para a promoção da aprendizagem.

Segundo as participantes, as oficinas psicoeducativas contribuíram para que elas pudessem aprender a lidar melhor com as próprias emoções e com as outras pessoas do seu convívio, proporcionaram crescimento e aprendizagem; ajudaram a enfrentar o medo de pensar; oportunizaram a aprendizagem sobre como agir em determinadas situações; promoveram o desenvolvimento de comportamentos mais assertivos como respeito aos próprios limites, importância de saber se posicionar e descoberta de melhores formas de se expressar; reconhecimento das necessidades e limitações do outro através do desenvolvimento da empatia; alertaram para o respeito às diferenças; orientaram sobre o desenvolvimento do autocontrole; incentivaram a busca pelo autoconhecimento e; contribuíram para as práticas profissionais. Silva e Silva (2009) também identificaram que a grande maioria dos professores participantes de sua pesquisa considerou que a educação emocional pode contribuir com as práticas docentes, indicando a necessidade da inserção dessa temática no currículo escolar.

A partir das considerações feitas pelas participantes pudemos constatar que o modelo de oficinas baseadas na Psicoeducação foi bastante adequado ao objetivo do projeto e para o público em questão estando de acordo com a literatura sobre o tema. 
Nogueira et al. (2017) identificaram pesquisas que utilizaram a psicoeducação como método de aprendizagem em grupo sobre melhores maneiras para enfrentar situações problemáticas ou geradoras de angústia aos participantes, tendo sido avaliadas como eficazes em suas propostas. Lemes e Ondere Neto (2017) apresentam estudos que reforçam a amplitude que a psicoeducação vem ganhando, destacando que esse método não se limita a um único espaço de atuação e que, além disso, vem sendo utilizado no enfrentamento do stress e da ansiedade, por exemplo.

As avaliações do grupo de extensão (segundo eixo) foram realizadas semanalmente, em momentos posteriores à realização das oficinas e também nos encontros para planejamento das atividades subsequentes. Durante as reuniões do grupo de extensão aconteciam discussões aprofundadas sobre as avaliações das participantes realizadas a cada encontro, avaliações sobre o funcionamento do grupo e sobre a pertinência dos temas trabalhados, além do compartilhamento das impressões pessoais e autoavaliações durante o processo. Nesses momentos, as extensionostas indicaram que o conhecimento sistemático e dialógico sobre as emoções lhes apresentou um novo olhar das possibilidades de aprendizagem sobre si e sobre como lidar com suas próprias questões, aquelas de ordem humana, que ultrapassam as barreiras da profissão, mas que, indiscutivelmente as fundamenta.

Essas primeiras categorias de percepções apresentadas pela experiência das extensionistas remontam à relação entre autoconhecimento e formação da identidade docente, ampliando a perspectiva das discussões sobre a relevância das emoções para o autodesenvolvimento integral dos sujeitos. Cardoso, Batista e Graça (2016) ressaltam a complexidade da formação da identidade, lembrando que ela se constitui a partir da indissociabilidade entre as dimensões individual e pessoal, elementos formadores também da identidade docente. No caso dessa última, Lopes (2007) aponta que a identidade profissional é constituinte da identidade social tanto quanto é dependente da identidade pessoal, sendo que esta última tem como unidade nuclear a experiência emocional dos sujeitos. Sendo assim, é possível compreender a relevância das emoções na constituição humana e sua relação com as repercussões comportamentais no contexto social.

Complementarmente, o processo como um todo foi percebido por elas como espaço de abertura de si e compartilhamento de experiências com o outro de maneira

Educação, Psicologia e Interfaces, Volume 3, Número3, p. 94-106, Setembro/Dezembro, 2019.

ISSN: 2594-5343. DOI: https://doi.org/10.37444/issn-2594-5343.v3i3.173 
Psicoeducação de professoras: contribuições da Teoria Cognitivo-Comportamental para promoção de saúde mental no contexto escolar

respeitável, num ambiente considerado seguro e confiável. Essencialmente, consideraram a experiência como capaz de promover aprendizagem e ampliar olhares, gerando nelas a vontade de conhecer mais. Para mim, professora, coordenadora e orientadora desse projeto, a experiência serviu para constatar algo que já vinha sendo percebido nas discussões realizadas em sala nas aulas de Psicologia da Educação: a necessidade de trabalhar nos(as) estudantes os aspectos subjacentes à formação profissional e essenciais à formação pessoal. Apesar de ter sido uma experiência nova, mostrou-se eficaz e, devido ao engajamento tanto das extensionistas quanto das participantes, obteve resultados além dos esperados.

\section{CONSIDERAÇÕES FINAIS}

A implementação desse projeto demonstrou que a proposta de trabalhar fatores que vão além da formação básica com professoras em formação, não somente é possível como também desejável. Encontramos através da realização das oficinas psicoeducativas uma maneira eficaz de aproximar os princípios da psicologia à educação de uma forma menos habitual, ainda incipiente, mas bastante promissora. A cada encontro fomos percebendo a necessidade de aprimoramento das atividades, no sentido de aprofundar discussões e práticas sobre determinados temas, assim como notamos o interesse de todas na permanência e ampliação do projeto.

Os resultados aqui apresentados fizeram com que, ao longo do processo e, especialmente ao final dele, tivéssemos a real dimensão da relevância desse projeto para a formação de professores (as), e nos fizeram refletir sobre a possibilidade de levar essa proposta reformulada para além dos espaços do CFP/UFRB, promovendo ações vinculadas também a escolas do município. Essas constatações nos levaram ao amadurecimento da proposta inicial, culminando na decisão de dar continuidade a esse projeto de extensão, ampliando suas possibilidades de atuação e promovendo ações mais efetivas e permanentes, visto que a formação docente deve ser pensada de maneira contínua e adaptada à realidade.

Nesse sentido as práticas realizadas através do referido projeto elucidaram a relevância de incluir a psicologia como área de estudo cotidiana na formação de professores, nos fazendo refletir sobre o quanto essa ciência ainda precisa expandir suas

Educação, Psicologia e Interfaces, Volume 3, Número3, p. 94-106, Setembro/Dezembro, 2019.

ISSN: 2594-5343. DOI: https://doi.org/10.37444/issn-2594-5343.v3i3.173 
ações. A TCC se mostrou uma área de conhecimento acessível e necessária ao desenvolvimento de habilidades para vida no contexto educacional, apesar de sua trajetória clínica. A proposta de trazer a Psicoeducação como recurso, oportunizou a aproximação entre as duas áreas de conhecimento numa perspectiva pouco conhecida no ambiente acadêmico, levando ao interesse de todas as participantes em aprofundar os conhecimentos adquiridos sobre como lidar com suas emoções, pensamentos e comportamentos, mostrando-se uma técnica acessível e de grande alcance.

Com base nessa experiência e, principalmente nos resultados obtidos através dela, sugerimos a elaboração de mais propostas de atuação da psicologia que saiam dos consultórios na intenção de realizar trabalhos in loco. Além disso, sentimos falta de embasamento teórico-prático voltado para o trabalho com grupos de professores, o que abre espaço para o desenvolvimento pesquisas com esses grupos que possam avaliar de maneira mais fidedigna o uso da TCC como recurso complementar à formação de professores e professoras.

\section{REFERÊNCIAS BIBLIOGRÁFICAS}

ALVES, J. M. (Org.). Abordagens cognitivo-comportamentais no contesto escolar. Novo Hamburgo: Sinopsys, 2018.

ANDRADE, P. S.; CARDOSO, T. A. O. Prazer e dor na docência: revisão bibliográfica sobre a Síndrome de Burnout. Saúde Soc., v. 21, n. 1, p. 129-140, 2012. Disponível em: http://www.scielo.br/pdf/sausoc/v21n1/13.pdf. Acesso em: 13 mar. 2019.

BAHLS, S. C.; NAVOLAR, A. B. B. Terapias cognitivo-comportamentais: conceitos e pressupostos teóricos. Revista Eletrônica de Psicologia, Curitiba, n. 04, p. 1-11, jul., 2004. Disponível em: http://files.personapsicologia.webnode.com/200000093024d10346f/Terapias\%20Cognitivo-comportamentais.pdf. Acesso em: 21 mar. 2019.

BECK, J. S. Introdução à Terapia Cognitivo-Comportamental. In: BECK, J. S.. Terapia Cognitivo-Comportamental: teoria e prática. 2 ed. Porto Alegre: Artmed, p. 21-36, 2013.

CAMINHA, R. M. Educar crianças: as bases de uma educação socioemocional um guia para pais, educadores e terapeutas. Novo Hamburgo: Sinopsys, 2014.

CARDOSO, M. I. S. T.; BATISTA, P. M. F.; GRAÇA, A. B. S. A identidade do professor: desafios colocados pela globalização. Revista Brasileira de Educação, v. 12, n. 65, p. 371-390. Disponível em: http://www.scielo.br/pdf/rbedu/v21n65/14132478-rbedu-21-65-0371.pdf . Acesso em: 29 set. 2019. 
CARLOTTO, M. S. Prevenção da síndrome de burnout em professores: um relato de experiência. Mudanças - Psicologia da Saúde, v. 11, n. 1, p. 31-39, 2014. Disponível em: https://www.metodista.br/revistas/revistas-

ims/index.php/MUD/article/view/4782/4383. Acesso em: 23 mai. 2018.

CASTRO, J. A. C. et al.. Prevalencia del síndrome de Burnout em los maestros.

Resultados de una investigación preliminar. Psiquiatria.com, v. 7, n. 1, 2003.

Disponível em: https://psiquiatria.com/estres/prevalencia-del-sindrome-de-burnout-enlos-maestros-resultados-de-una-investigacion-preliminar/. Acesso em: 13 mar. 2019.

CRUZ, R. M. et al.. Saúde docente, condições de carga de trabalho. Revista Eletrônica de Investigação y Docência (REID), v. 4, Julio, p. 147-160, 2010. Disponível em: http://www.ujaen.es/revista/reid/revista/n4/REID4art8.pdf. Acesso em: 12 Mar. 2019.

GOULART, I. B. Psicologia da educação: Fundamentos teóricos, aplicações à prática pedagógica. 6. ed. Petrópolis: Editora Vozes, 1999.

LEMES, C; ONDERE NETO, J. Aplicações da psicoeducação no contexto da saúde. Temas em Psicologia, v. 25, n. 1, p. 17-28, 2017. Disponível em: http://pepsic.bvsalud.org/pdf/tp/v25n1/v25n1a02.pdf. Acesso em: 04 Jan. 2019.

LOPES, A. Laconstrucción de identidades docentes como constructo de estructura y dinâmica sistêmicas: argumentación y vurtualidades teóricas y prácticas. Revista de Currículum y Formación de Professorado, v. 11, n. 3, p. 1-25. Disponível em: https://www.redalyc.org/pdf/567/56711307.pdf . Acesso em: 29 set. 2019.

MELLO, R. B. P. F.; CORREIA JUNIOR, A. M. As crenças do educador podem contribuir para o sucesso educacional? Em: ALVES, J. M. (Org.). Abordagens Cognitivo Comportamentais no contexto escolar. Novo Hamburgo: Sinopsys, 2018.

NOGUEIRA, C. A. et al.. A importância da Psicoeducação na terapia cognitivocomportamental: uma revisão sistemática. Revista das Ciências da Saúde do Oeste Baiano, Higia, v. 12, n. 1, p. 108-120, 2017. Disponível em: http://www.fasb.edu.br/revista/index.php/higia/article/view/190/211. Acesso em: 08 Mai. 2019.

OMS (Portugal). Integração da saúde mental nos cuidados de saúde primários: Uma perspectiva global. Lisboa: Ministério da Saúde, 2008.

SILVA, A. C. R.; SILVA, G. A. A educação emocional e o preparo do profissional docente. In: Atas do X Congresso Internacional Galego-Português de Psicopedagogia. Braga: Universidade do Minho, p. 9-11, 2009. Disponível em: https://studylib.es/doc/6460070/a-educa\%C3\%A7\%C3\%A3o-emocional-e-o-preparodo-profissional-docente. Acesso em: 14 Mai. 2019.

SILVA, G. G. J. et al. Considerações sobre o transtorno depressivo no trabalho. Revista Brasileira de Saúde Ocupacional, São Paulo, v. 34, n. 119, p. 79-87, 2009. 
Disponível em: https://www.redalyc.org/pdf/1005/100512332009.pdf. Acesso em: 21 mar. 2019.

SILVA, M. E. P. Burnout: por que sofrem os professores? Estudos e pesquisas em psicologia, ano 6, n. 1, p. 89-98, 2006. Disponível em: http://www.redalyc.org/articulo.oa?id=451844611009. Acesso em: 23 Mai. 2019.

VERCELLI, L. C. A. A psicologia da educação na formação docente. Dialogia, São Paulo, v. 7, n. 2, p. 223-233, 2008. Disponível em:

http://periodicos.uninove.br/index.php?journal=dialogia\&page=article\&op=view\&path \%5B\%5D=1016\&path\%5B\%5D=1538. Acesso em: 13 Dez 2018.

WRIGHT, J. H.; BASCO, M. R.; THASE, M. E. Aprendendo a terapia cognitivocomportamental: um guia ilustrado. Porto Alegre: Artmed, 2008.

\section{Credenciais da/os autora/es:}

GOMES, Sabrina Torres. Mestrado em Psicologia do Desenvolvimento pela UFBA e doutorado na linha de Infância e Contextos Culturais - Desenvolvimento Humano pela UFBA. Professora Adjunta da Universidade Federal do Recôncavo/Brasil E-mail: sabrinagomes@ufrb.edu.br

JESUS, Fernanda Conceição de. Universidade Federal do Recôncavo da Bahia/Brasil E-mail: nandaconceicao31@gmail.com

JESUS, Lusimara Gonçalves Miranda da Silva. Universidade Federal do Recôncavo da Bahia/Brasil E-mail: ugagoncalves@gmail.com

\section{Endereço para correspondência:}

Como citar este artigo (Formato ABNT): GOMES, Sabrina Torres; JESUS, Fernanda Conceição de; JESUS, Lusimara Gonçalves Miranda da Silva. Psicoeducação de professoras: contribuições da Teoria Cognitivo-Comportamental para promoção de saúde mental no contexto escolar. Educação, Psicologia e Interfaces, v. 3, n.3, p. 94-106, 2019. DOI: https://doi.org/10.37444/issn-2594-5343.v3i3.173

Recebido: 07/08/2019.

Aceito: 20/11/2019. 